QUARTIERO A; FARIA MV; RESENDE JTV; FIGUEIREDO AST; CAMARGO LKP; SANTOS RL; KOBORI RF. 2014. Desempenho agronômico, heterose e estabilidade fenotípica de genótipos de cebola. Horticultura Brasileira 32: 259-266. DOI http://dx.doi.org/10.1590/S0102-05362014000300004

\title{
Desempenho agronômico, heterose e estabilidade fenotípica de genótipos de cebola
}

\author{
Anderson Quartiero ${ }^{1}$; Marcos V Faria ${ }^{1}$; Juliano TV Resende ${ }^{1}$; Alex ST Figueiredo ${ }^{1}$; Letícia KP Camargo ${ }^{1}$; \\ Ricardo L Santos'; ${ }^{2}$ Rômulo F Kobori² \\ ${ }^{1}$ UNICENTRO, Depto. Agronomia, C. Postal 3010, 85040-080 Guarapuava-PR; andersonquartiero@ig.com.br; mfaria@unicentro.br; \\ jresende@unicentro.br; alexfigueiredo@hotmail.com; leti_kpc@yahoo.com.br; ${ }^{2}$ Sakata Seed Sudamerica, C. Postal 427, 12906-840 \\ Bragança Paulista-SP; ricardo.lima@sakata.com.br; romulo.kobori@sakata.com.br
}

\section{RESUMO}

O objetivo do trabalho foi avaliar o desempenho agronômico, a heterose e a estabilidade fenotípica de 50 genótipos de cebola (uma linhagem e oito híbridos experimentais do grupo Baia, 10 linhagens e 22 híbridos experimentais do grupo Crioula, seis variedades de polinização aberta e três híbridos comerciais) em três épocas de cultivo em Guarapuava-PR, na safra 2010/2011. Três experimentos foram conduzidos no delineamento em blocos casualizados, com três repetições. As mudas com 45 dias de idade foram transplantadas em 27 de maio, 30 de junho e 03 de agosto. Foram avaliadas características agronômicas dos bulbos. Para a estabilidade fenotípica dos genótipos foi feita a análise pelo método de Annicchiarico. Foi estimada a heterose de sete híbridos. A primeira época proporcionou os maiores valores de massa média e a maior produtividade de bulbos; a terceira época proporcionou as médias mais baixas. Para a produtividade de bulbos, apenas $26 \%$ dos genótipos apresentaram índice de confiança geral $\left(\omega_{\mathrm{i}}\right)$ acima de $100 \%$. Os híbridos experimentais HB1266, HB2285, HC3119, HC3126, HC3479 e HC3488 apresentaram os maiores índices de confiança para a produtividade, embora na primeira época não tenham participado do grupo dos genótipos mais produtivos. A heterose média observada para a massa média dos bulbos foi de 38\%, com variação de 16\% (HC3485) a 63\% (híbrido $\mathrm{HC} 3483$ ) e para a produtividade foi de $36 \%$, variando de $16 \%$ (HC3485) a $57 \%(\mathrm{HC} 3483)$.

Palavras-chave: Allium cepa, interação genótipos x ambientes, índice de confiança de Annicchiarico.

\section{ABSTRACT}

Agronomic performance, heterosis and phenotypic stability of onion genotypes

The objective of this study was to evaluate the performance, heterosis and phenotypic stability of 50 genotypes of onion (a lineage and eight experimental hybrids of Baia group, 10 lineages and 22 experimental hybrids of Creole group, six open pollinated varieties and three commercial hybrids) in three crop seasons in Guarapuava, Paraná state, Brazil, in 2010/2011. Three experiments were carried out in randomized block design with three replications. Seedlings aging 45 days were transplanted on May 27, June 30 and August 03. We evaluated agronomic characteristics of bulbs. For the analysis of phenotypic stability of genotypes the method of Annicchiarico was used. We also estimated the heterosis of seven hybrids. The first period provided the highest values of average mass and increased yield of bulbs and the third period provided the lowest values. Only $26 \%$ of genotypes showed general confidence index $\left(\omega_{i}\right)$ above $100 \%$ for bulb yield. The experimental hybrids HB1266, HB2285, HC3119, HC3126, HC3479 and HC3488 showed the highest confidence index for bulbs yield, although in the first period they did not participate in the group of highest yield genotypes. The mean of heterosis observed for the average mass of bulbs was 38\%, ranging from $16 \%$ ( $\mathrm{HC} 3485)$ to $63 \%$ (hybrid $\mathrm{HC} 3483$ ) and for yield was $36 \%$, ranging from $16 \%$ (HC3485) to $57 \%$ (HC3483).

Keywords: Allium cepa, genotypes x environments interaction, confidence index of Annicchiarico.

\section{(Recebido para publicação em 13 de janeiro de 2014; aceito em 9 de maio de 2014) (Received on January 13, 2014; accepted on May 9, 2014)}

\begin{abstract}
A cebola (Allium cepa) é a terceira hortaliça em importância econômica no mundo e a terceira mais produzida no Brasil (Agrianual, 2011). Em 2013 a safra brasileira de cebola foi de 1.314.701 toneladas de bulbos, em 57.341 hectares de área plantada, proporcionando produtividade média de 22,29 t ha-1. Em 2013, no estado do Paraná foram produzidas 153.342 toneladas, em área de 7.449 hectares, com produtividade média de $21,94 \mathrm{t}$ $\mathrm{ha}^{-1}$, sendo responsável por $11,7 \%$ de toda cebola produzida no Brasil (IBGE, 2013).
\end{abstract}

A cebola é uma hortaliça cuja adaptação em determinada localidade é condicionada por fatores ambientais, notadamente o fotoperíodo e a temperatura, o que limita a recomendação de uma mesma cultivar para uma faixa ampla de latitudes. No mesmo local o fotoperíodo e a temperatura se alteram em função da época do ano e, se as condições não satisfizerem as exigências da cultura, pode haver perdas na produção, com redução da bulbificação, emissão precoce do pendão floral, ocorrência de plantas improdutivas denominadas de "charutos" e de pequenos bulbos (Resende et al., 2003).

A exploração da heterose tem sido o princípio usado no melhoramento e produção de muitas hortaliças (Maluf, 2001). A procura por híbridos de cebola mais produtivos tem aumentado em função da superioridade agronômica desses em relação às cultivares de polinização livre (May et al., 2007). Assim, informações sobre o desempenho dos híbridos e das respectivas linhagens parentais 
são importantes na determinação dos melhores genótipos a fim de se obter maiores ganhos heteróticos.

Um dos fatores que limita a produtividade da cebola no Paraná, e mais especificamente na região centro-sul do estado, é a escassez de informações sobre o comportamento e a adaptação de genótipos nas condições edafoclimáticas locais, considerando as variações no manejo e na época de cultivo. Essas informações precisam ser consideradas nos programas de melhoramento, para que se obtenha sucesso no desenvolvimento de novos híbridos. Assim, devido à grande demanda por genótipos produtivos e adaptados às condições climáticas paranaenses, propôs-se avaliar a estabilidade, a heterose e o desempenho agronômico de genótipos experimentais de cebola cultivados em diferentes épocas em Guarapuava-PR.

\section{MATERIAL E MÉTODOS}

Três experimentos foram realizados no ano de 2010 nas dependências da Universidade Estadual do Centro-Oeste em Guarapuava-PR (25 23'36"s, $51^{\circ} 27^{\prime} 9$ 'O , altitude $\left.1.025 \mathrm{~m}\right)$. O clima da região é classificado como subtropical úmido mesotérmico $(\mathrm{Cfb})$ sem estação seca definida e com verões pouco quentes e invernos rigorosos, de acordo com a classificação de Köppen. O solo da área experimental foi classificado como Latossolo Bruno Distroférrico típico, de textura argilosa.

Foram avaliados 50 genótipos de cebola de base genética diferente, sendo 1 linhagem do grupo Baia (LB) e 8 híbridos experimentais do grupo Baia (HB), 10 linhagens do grupo Criolula (LC) e 22 híbridos experimentais do grupo Crioula (HC), 6 variedades de polinização aberta (OP) e 3 híbridos comerciais cultivados na região utilizados como testemunhas (Buccanneer, Optima e Perfecta). Os genótipos experimentais são propriedade da empresa Sakata Seeds Sudamerica Ltda. e são derivados de progênies desenvolvidas especificamente para a região sul do país, a partir de germoplasma nacional denominado Super Precoce e Crioula.

As mudas foram produzidas em bandejas de isopor do tipo "speedling" com 200 células, preenchidas com substrato comercial Plantmax ${ }^{\circledR}$ e mantidas em casa de vegetação com irrigação por aspersão. Após a emergência das plantas, foi feita aplicação semanal de fertilizante foliar Yogen ${ }^{\circledR}(30 \% \mathrm{~N} ; 10 \%$ $\mathrm{P}_{2} \mathrm{O}_{5} ; 10 \% \mathrm{~K}_{2} \mathrm{O} ; 0,10 \% \mathrm{MgO} ; 0,05 \%$ $\mathrm{B}_{2} \mathrm{O}_{3} ; 0,10 \% \mathrm{MnO}$ e $0,05 \% \mathrm{Zn}$ ) na dose recomendada pelo fabricante.

Os 50 tratamentos foram avaliados em três épocas de cultivo, correspondentes aos transplantios realizados em 27 de maio, 30 de junho e 03 de agosto. Em cada época as mudas foram transplantadas aos 45 dias após a semeadura.

Em cada época foi conduzido um experimento em delineamento de blocos casualizados com três repetições, totalizando 150 parcelas. Cada parcela possuía área útil de $1,5 \mathrm{~m}^{2}(1,0 \times 1,5$ m) com 100 plantas distribuídas em 10 linhas, com espaçamento de 0,15 $\mathrm{m}$ entre linhas e $0,10 \mathrm{~m}$ entre plantas, com população equivalente a $660 \mathrm{mil}$ plantas ha' ${ }^{-1}$.

A correção do solo da área experimental e as adubações de plantio e de cobertura foram realizadas com base na análise do solo, seguindo as recomendações de Filgueira (2005). Foi realizado o controle químico de pragas, doenças e plantas daninhas por meio da aplicação de inseticidas, fungicidas e herbicidas recomendados para a cultura. As irrigações foram por aspersão, seguindo as recomendações de Marouelli et al. (2005).

A colheita foi realizada após o estalo das plantas, ou seja, quando a parte aérea apresentava-se tombada rente ao solo. Nessa fase foi interrompida a irrigação. As datas das colheitas das plantas foram 08/11/2010, 15/12/2010 e 17/01/2011 para a primeira, segunda e terceira época, respectivamente.

Em cada experimento, após a cura, os bulbos de cada parcela foram contados e pesados. Foi avaliada a produtividade de bulbos ( $\left.\mathrm{t} \mathrm{ha}^{-1}\right)$ e a massa média dos bulbos (g bulbo ${ }^{-1}$ ).

Foi avaliada a estabilidade dos 50 genótipos quanto à produtividade de bulbos, considerando as três épocas, pelo método de Annicchiarico (1992). Foi estimado o índice de confiabilidade geral $\left(\omega_{\mathrm{i}}\right)$ dos genótipos em valores percentuais, que determina o risco de adoção de determinado genótipo em relação à média dos demais genótipos utilizados nos diferentes ambientes (Souza et al., 2013). Esse método possibilita a identificação dos genótipos que apresentam comportamento estável à medida que se altera o ambiente de produção. Quanto maior o valor de $\omega_{i}$ acima de $100 \%$, maior é a confiabilidade e chance de sucesso na escolha de um determinado genótipo para os diferentes ambientes avaliados (Cruz \& Carneiro, 2006). Para esta análise utilizou-se o software computacional Estabilidade (Ferreira, 2000).

Estimativas da heterose $(\mathrm{H})$ foram obtidas de sete híbridos que possuíam as duas linhagens parentais também avaliadas no experimento: LC2873A $\mathrm{x}$ LC3068-14 = HC3490; LC3031A $x$ LC3068-3 = HC3489; LC3031A $\mathrm{x}$ LC3153 = HC3485; LC3031A x LC3 $156=$ HC3480; LC3031A $x$ LC3157=HC3486; LC2873A x LC3153 $=\mathrm{HC} 3483$; LC2873A $\mathrm{x}$ LC3156 = HC3477. Foi utilizada a expressão $\mathrm{H}=\mathrm{F}_{1}-\left(\mathrm{P}_{1}+\mathrm{P}_{2}\right) / 2$, em que: $\mathrm{F}_{1}=$ média da primeira geração oriunda do cruzamento (híbrido); $\mathrm{P}_{1}=$ média da linhagem parental $1 ; \mathrm{P}_{2}=$ média da linhagem parental 2 . Foram considerados os valores médios das três épocas de cultivo.

Os dados referentes à produtividade de bulbos foram submetidos às análises de variância individuais e posteriormente, depois de verificada a homocedasticidade, foi realizada a análise conjunta e as médias dos tratamentos foram agrupadas pelo teste de Scott \& Knott a $5 \%$ de probabilidade, utilizando-se o software computacional Genes (Cruz, 2007).

\section{RESULTADOS E DISCUSSÃO}

Houve diferenças significativas entre os genótipos e entre as épocas de cultivo, tanto para a massa média quanto para a produtividade de bulbos. Também houve efeito significativo da interação genótipos x épocas, reflexo da forte dependência da cebola ao fotoperíodo e temperatura para bulbificação e interação com outros fatores ambientais, requerendo programas de 
Tabela 1. Massa média $\left(\mathrm{g}\right.$ bulbo $\left.{ }^{-1}\right)$ de 50 genótipos de cebola cultivados em três épocas [average mass $\left(\mathrm{g}\right.$ bulb $\left.{ }^{-1}\right)$ of 50 onion genotypes grown in three seasons]. Guarapuava, UNICENTRO, 2011.

\begin{tabular}{|c|c|c|c|c|c|c|c|c|c|}
\hline Genótipo & $1^{a}$ época & $2^{\mathrm{a}}$ época & $3^{\mathrm{a}}$ época & Genótipo & $1^{a}$ época & $2^{\text {a }}$ époc & oca & $3^{\text {a época }}$ & \\
\hline HB1266 & $138,24 \mathrm{c}^{*} \mathrm{~A}$ & 101,44 a $\mathrm{B}$ & 65,26 a $C$ & HC3487 & 85,06 e $A$ & $53,05 \mathrm{c}$ & c $\quad$ B & 61,42 a & $\bar{B}$ \\
\hline HB1555 & $156,80 \mathrm{~b} \mathrm{~A}$ & 51,48 с B & 51,85 b B & HC3488 & 115,37 c A & $74,27 \mathrm{~b}$ & b $\quad B$ & 84,06 a & B \\
\hline HB2285 & 149,18 b A & 90,41 a $B$ & 64,18 a $\mathrm{C}$ & HC3489 & 129,52 с A & $86,02 \mathrm{~b}$ & b $\quad B$ & $50,71 \mathrm{~b}$ & $\mathrm{C}$ \\
\hline HB2572 & $131,10 \mathrm{c} \mathrm{A}$ & 86,31 b B & 40,48 b C & HC3490 & $106,10 \mathrm{~d} \mathrm{~A}$ & 90,29 a & A & $53,38 \quad \mathrm{~b}$ & B \\
\hline HB2574 & $109,85 \mathrm{~d} \mathrm{~A}$ & 83,01 b B & 66,93 a $B$ & НC3491 & 84,31 e $A$ & $100,82 \mathrm{a}$ & A & 72,30 a & A \\
\hline HB2575 & $128,48 \mathrm{c} \mathrm{A}$ & $74,50 \mathrm{~b} \mathrm{~B}$ & 43,73 b C & Perfecta & 182,20 a $\mathrm{A}$ & $87,85 \mathrm{~b}$ & b $\quad B$ & $48,11 \quad b$ & $\mathrm{C}$ \\
\hline HB2578 & $104,24 \mathrm{~d} \mathrm{~A}$ & 58,66 с B & 64,92 a $B$ & Buccanneer & 170,85 a $\mathrm{A}$ & $72,94 \mathrm{~b}$ & b $\quad B$ & 81,66 a & B \\
\hline HB3451 & $96,92 \mathrm{~d} A$ & $75,58 \mathrm{~b} \mathrm{~A}$ & $40,29 \mathrm{~b} \quad \mathrm{~B}$ & Optima & 171,28 a $\mathrm{A}$ & $68,78 \mathrm{c}$ & c $\mathrm{B}$ & $51,56 \mathrm{~b}$ & B \\
\hline HC3119 & $110,85 \mathrm{~d} \mathrm{~A}$ & 96,57 a $\mathrm{A}$ & 70,74 a $B$ & LB2982 & $115,95 \mathrm{c} \mathrm{A}$ & $46,92 \mathrm{~d}$ & d $\quad B$ & $51,24 \mathrm{~b}$ & B \\
\hline HC3120 & $109,60 \mathrm{~d} A$ & 106,48 a $\mathrm{A}$ & $58,25 \mathrm{~b}$ B & LC2873A & 74,48 e $\mathrm{A}$ & $41,02 \mathrm{~d}$ & d $\quad B$ & $23,14 \mathrm{~b}$ & B \\
\hline HC3121 & $110,23 \mathrm{dA}$ & 66,00 с B & 66,77 a $B$ & LC2951 & $91,46 \mathrm{~d} \mathrm{~A}$ & $67,34 \mathrm{c}$ & A & 73,16 a & A \\
\hline HC3123 & $116,37 \mathrm{c} \mathrm{A}$ & 56,81 с B & 72,39 a $B$ & & & $65,72 \mathrm{c}$ & c $\mathrm{B}$ & $43,82 \mathrm{~b}$ & B \\
\hline HC3124 & $103,94 \mathrm{~d} \mathrm{~A}$ & $65,34 \mathrm{c} \mathrm{B}$ & 73,69 a $B$ & LC3068-14 & 78,77 e $\mathrm{A}$ & $82,28 \quad b$ & A & $53,13 \mathrm{~b}$ & B \\
\hline HC3125 & 115,42 c A & 75,05 b B & 88,48 a $\mathrm{B}$ & LC3068-3 & 84,03 e $A$ & $66,60 \mathrm{c}$ & A & $41,91 \mathrm{~b}$ & B \\
\hline HC3126 & 120,87 c A & 85,10 b B & 61,97 a $B$ & LC3153 & 55,48 e $\mathrm{A}$ & $28,49 \mathrm{~d}$ & d A & $36,08 \mathrm{~b}$ & A \\
\hline HC3477 & $107,33 \mathrm{~d} \mathrm{~A}$ & 56,77 с B & $46,25 \mathrm{~b} \quad \mathrm{~B}$ & LC3154 & 85,74 e $\mathrm{A}$ & $40,39 \mathrm{~d}$ & d $\quad B$ & $47,05 \mathrm{~b}$ & B \\
\hline HC3478 & $102,94 \mathrm{~d} \mathrm{~A}$ & 55,77 с $\mathrm{B}$ & 64,42 a $B$ & LC3156 & 75,46 e $\mathrm{A}$ & $46,24 \mathrm{~d}$ & d $\quad B$ & $41,96 \mathrm{~b}$ & B \\
\hline HC3479 & $113,58 \mathrm{c} \mathrm{A}$ & 72,83 b B & 62,61 a $B$ & LC3157 & 78,21 e $\mathrm{A}$ & $41,37 \mathrm{~d}$ & B & $38,89 \mathrm{~b}$ & B \\
\hline HC3480 & $102,30 \mathrm{dA}$ & 76,43 b B & $55,04 \mathrm{~b}$ B & LC3291A & 69,21 e $A$ & $31,70 \mathrm{~d}$ & B & $57,30 \mathrm{~b}$ & $\mathrm{~A}$ \\
\hline HC3481 & $106,66 \mathrm{~d} \mathrm{~A}$ & 95,13 a $\mathrm{A}$ & 38,53 b B & OP2751 & $127,28 \mathrm{c} \mathrm{A}$ & $82,75 \mathrm{~b}$ & B & 75,96 a & B \\
\hline HC3482 & 136,65 с A & 108,68 a $\mathrm{B}$ & 53,60 b C & OP2753 & $100,94 \mathrm{~d} \mathrm{~A}$ & $66,60 \mathrm{c}$ & B & 67,73 a & B \\
\hline HC3483 & $95,43 \mathrm{~d} A$ & 50,88 с B & 56,83 b B & OP2930 & $109,17 \mathrm{~d} \mathrm{~A}$ & $76,03 \mathrm{~b}$ & B & $45,09 \mathrm{~b}$ & $\mathrm{C}$ \\
\hline HC3484 & 63,41 e $A$ & 60,40 с A & 70,05 a $\mathrm{A}$ & OP2931 & $121,52 \mathrm{c} \mathrm{A}$ & 89,42 a & B & 67,33 a & B \\
\hline HC3485 & 82,96 e A & 54,02 с B & 50,45 b B & OP3211 & 120,76 с A & $79,86 \mathrm{~b}$ & b $\quad B$ & $54,42 \mathrm{~b}$ & $\mathrm{C}$ \\
\hline HC3486 & $122,23 \mathrm{c} \mathrm{A}$ & $73,94 \mathrm{~b} \mathrm{~B}$ & 56,45 b B & OP3212 & $123,21 \mathrm{c} \mathrm{A}$ & $58,91 \quad \mathrm{c}$ & $\mathrm{C}$ & 84,07 a & $\mathrm{B}$ \\
\hline Média & & & & & $109,67 \mathrm{~A}$ & $70,46 \mathrm{~B}$ & & $57,79 \mathrm{C}$ & \\
\hline
\end{tabular}

${ }^{*}$ Médias seguidas pela mesma letra minúscula na coluna e maiúscula na linha pertencem ao mesmo grupo pelo teste de Scott-Knott, a $5 \%$ de probabilidade.(means followed by the same lowercase and uppercase letters in column and line, respectively, belong to the same group by the Scott-Knott test, $5 \%$ ).

melhoramento para latitudes, regiões ou épocas específicas (Santos et al., 2004; Leite et al., 2009).

Ocorreu redução significativa no tamanho médio dos bulbos à medida que se atrasou o plantio (Tabela 1). O atraso em relação à época ideal para o cultivo de cada genótipo promove redução significativa das características relacionadas à produtividade de bulbos, dentre elas a massa média. Essa redução ocorre devido à bulbificação precoce das plantas, em função das condições de fotoperíodo e temperatura (Sirtoli et al., 2010).

Foram formados cinco grupos de genótipos na primeira época de cultivo, quatro grupos na segunda época e apenas dois grupos na terceira época quanto à massa média dos bulbos. Con- siderando apenas os grupos de maiores médias, em cada uma das três épocas, a variação na amplitude dos valores foi de 182,20 g (Perfecta) a 170,85 g (Buccanneer) para o grupo superior da primeira época, 108,68 g (HC3482) a $89,42 \mathrm{~g}$ (OP2931) para a segunda época e $88,48 \mathrm{~g}(\mathrm{HC} 3125)$ a $61,42 \mathrm{~g}(\mathrm{HC} 3487)$ para a terceira.

Na primeira época os três híbridos comerciais formaram o grupo de genótipos de maior massa média, superarando estatisticamente todos os genótipos experimentais. Em contrapartida, 12 genótipos apresentaram bulbos com peso médio inferior a $86,0 \mathrm{~g}$, sendo quatro híbridos experimentais (HC3484, HC3485, HC3487 e HC3491) e oito linhagens experimentais (LC2873A, LC3068-14, LC3068-3, LC3153, LC3154,
LC3156, LC3157 e LC3291A). Esses genótipos experimentais, todos pertencentes ao grupo Crioula, mostraram-se mais adaptados a condições de cultivo mais tardias quando comparados com os híbridos comerciais mais cultivados na região, que tiveram melhor desempenho na primeira época de plantio.

Para a segunda época de cultivo, nove genótipos formaram o grupo de maior massa média de bulbos, sendo dois híbridos Baia (HB1266 e HB2285), seis híbridos Crioula (HC3119, HC3120, HC3481, HC3482, HC3490 e HC3491) e a variedade OP2931 (Tabela 1). A diferença de tempo entre a primeira e a segunda época propiciou redução significativa no tamanho médio dos bulbos dos híbridos comerciais Buccanneer, 
Tabela 2. Produtividade $\left(\mathrm{t} \mathrm{ha}^{-1}\right)$ de 50 genótipos de cebola cultivados em três épocas [yield $\left(\mathrm{t} \mathrm{ha}^{-1}\right)$ of 50 onion genotypes grown in three seasons]. Guarapuava, UNICENTRO, 2011.

\begin{tabular}{|c|c|c|c|c|c|c|c|c|c|c|c|c|c|}
\hline \multirow{3}{*}{$\begin{array}{l}\text { Genótipo } \\
\text { HB1266 }\end{array}$} & \multicolumn{6}{|c|}{ Produtividade $\left(\mathrm{t} \mathrm{ha}^{-1}\right)$} & \multirow{3}{*}{$\begin{array}{l}\text { Genótipo } \\
\text { HC3487 }\end{array}$} & \multicolumn{6}{|c|}{ Produtividade (t ha-1) } \\
\hline & \multicolumn{2}{|c|}{$1^{\mathrm{a}}$ época } & \multicolumn{2}{|c|}{$2^{\mathrm{a}}$ época } & \multicolumn{2}{|c|}{$3^{\mathrm{a}}$ época } & & \multicolumn{2}{|c|}{$1^{\mathrm{a}}$ época } & \multicolumn{2}{|c|}{$2^{\mathrm{a}}$ época } & \multicolumn{2}{|c|}{$3^{\mathrm{a}}$ época } \\
\hline & 82,9 & ${ }^{*} \mathrm{c} \mathrm{A}$ & 60,9 & a B & 39,2 & $\mathrm{aC}$ & & 51,0 & e A & 31,8 & c B & 36,9 a & $B$ \\
\hline HB1555 & 94,1 & b A & 30,9 & c B & 31,1 & $\mathrm{~b} B$ & HC3488 & 69,2 & $\mathrm{~d} \mathrm{~A}$ & 44,6 & b B & 50,4 a & $B$ \\
\hline HB2285 & 89,5 & b A & 54,2 & a B & 38,5 & a $\mathrm{C}$ & HC3489 & 77,7 & c A & 51,6 & b B & $30,4 \mathrm{~b}$ & $\mathrm{C}$ \\
\hline HB2572 & 92,0 & $\mathrm{~b} A$ & 51,8 & $\mathrm{~b} B$ & 24,3 & $\mathrm{~b} \mathrm{C}$ & НC3490 & 63,7 & $\mathrm{~d} A$ & 54,2 & a A & $32,0 \mathrm{~b}$ & $\mathrm{~B}$ \\
\hline HB2574 & 65,9 & $\mathrm{~d} A$ & 49,8 & b B & 40,2 & a B & НC3491 & 50,6 & e A & 60,5 & a $\mathrm{A}$ & $43,4 \quad \mathrm{a}$ & $\mathrm{A}$ \\
\hline HB2575 & 77,1 & c A & 44,7 & $\mathrm{~b} B$ & 26,2 & $\mathrm{~b} C$ & Perfecta & 109,3 & a $\mathrm{A}$ & 52,7 & b B & $28,9 \mathrm{~b}$ & $\mathrm{C}$ \\
\hline HB2578 & 62,5 & $\mathrm{~d} A$ & 35,2 & c B & 39,0 & a B & Buccanneer & 102,5 & a $\mathrm{A}$ & 43,8 & b B & $49,0 \quad \mathrm{a}$ & $B$ \\
\hline HB3451 & 58,2 & $\mathrm{~d} A$ & 45,4 & $\mathrm{~b} A$ & 24,2 & b B & Optima & 102,8 & a $\mathrm{A}$ & 41,3 & c B & $31,0 \quad b$ & B \\
\hline HC3119 & 66,5 & $\mathrm{~d} A$ & 57,9 & $\mathrm{a} A$ & 42,4 & a B & LB2982 & 69,6 & $\mathrm{~d} A$ & 28,2 & d B & $30,7 \quad b$ & B \\
\hline HC3120 & 65,8 & $\mathrm{~d} A$ & 63,9 & a $\mathrm{A}$ & 35,1 & $\mathrm{~b} B$ & LC2873A & 44,7 & e $A$ & 24,6 & d B & $13,9 \mathrm{~b}$ & B \\
\hline HC3121 & 66,1 & $\mathrm{~d} A$ & 39,6 & c B & 40,1 & a B & LC2951 & 54,9 & e A & 40,4 & c A & 43,9 a & $\mathrm{A}$ \\
\hline HC3123 & 69,8 & $\mathrm{~d} A$ & 34,1 & c B & 43,4 & a B & LC3031A & 55,0 & e $\mathrm{A}$ & 39,4 & c B & $26,3 \mathrm{~b}$ & $\mathrm{~B}$ \\
\hline HC3124 & 62,4 & d A & 39,2 & c B & 44,2 & a B & LC3068-14 & 47,3 & e $A$ & 49,4 & b A & $31,9 \quad \mathrm{~b}$ & $\mathrm{~B}$ \\
\hline HC3125 & 69,3 & $\mathrm{~d} A$ & 45,0 & $b$ B & 53,1 & a B & LC3068-3 & 50,4 & e A & 40,0 & c A & $25,2 \mathrm{~b}$ & B \\
\hline HC3126 & 72,5 & c A & 51,1 & $\mathrm{~b} B$ & 37,2 & a $B$ & LC3153 & 33,3 & e $A$ & 17,1 & d A & $21,6 \mathrm{~b}$ & $\mathrm{~A}$ \\
\hline HC3477 & 64,4 & $\mathrm{~d} A$ & 34,1 & c B & 27,8 & b B & LC3154 & 51,4 & e A & 24,2 & d B & $28,2 \mathrm{~b}$ & B \\
\hline HC3478 & 61,8 & $\mathrm{~d} A$ & 33,5 & c B & 38,7 & a B & LC3156 & 45,3 & e $\mathrm{A}$ & 27,7 & d B & $25,2 \mathrm{~b}$ & B \\
\hline HC3479 & 68,1 & $\mathrm{~d} A$ & 43,7 & b B & 37,6 & a B & LC3157 & 46,9 & e $A$ & 24,8 & d B & $23,3 \mathrm{~b}$ & B \\
\hline HC3480 & 61,4 & $\mathrm{~d} A$ & 45,9 & $\mathrm{~b} B$ & 33,0 & $\mathrm{~b} B$ & LC3291A & 41,5 & e $A$ & 19,0 & d B & $34,4 \mathrm{~b}$ & $\mathrm{~A}$ \\
\hline HC3481 & 64,0 & $\mathrm{~d} A$ & 57,1 & a $\mathrm{A}$ & 23,1 & $\mathrm{~b} B$ & OP2751 & 76,4 & c A & 49,7 & b B & 45,5 a & $B$ \\
\hline HC3482 & 82,0 & c A & 65,2 & a B & 32,2 & $\mathrm{~b} \mathrm{C}$ & OP2753 & 60,6 & $\mathrm{~d} A$ & 40,0 & c B & 40,6 a & $B$ \\
\hline HC3483 & 57,3 & $\mathrm{~d} A$ & 30,5 & c B & 34,1 & b B & OP2930 & 65,5 & $\mathrm{~d} A$ & 45,6 & b B & $27,1 \quad b$ & $\mathrm{C}$ \\
\hline HC3484 & 38,0 & e $\mathrm{A}$ & 36,2 & c A & 42,0 & $\mathrm{a} A$ & OP2931 & 72,9 & c A & 53,7 & a B & $40,4 \quad \mathrm{a}$ & $B$ \\
\hline HC3485 & 49,8 & e $A$ & 32,4 & c B & 30,3 & $\mathrm{~b} B$ & OP3211 & 72,5 & c A & 47,9 & b B & $32,6 \mathrm{~b}$ & $\mathrm{C}$ \\
\hline HC3486 & 73,3 & c A & 44,4 & $\mathrm{~b} B$ & 33,9 & $\mathrm{bB}$ & OP3212 & 73,9 & c A & 35,3 & $\mathrm{c} \mathrm{C}$ & 50,4 a & $\mathrm{B}$ \\
\hline Média & & & & & & & & 66,07 & & 42,27 & $9 \mathrm{~B}$ & $34,67 \mathrm{C}$ & \\
\hline
\end{tabular}

${ }^{*}$ Médias seguidas pela mesma letra minúscula na coluna e maiúscula na linha pertencem ao mesmo grupo pelo teste de Scott-Knott, a 5\% de probabilidade (means followed by the same lowercase and uppercase letters in column and line, respectively belong to the same group by the Scott-Knott test $5 \%$ ).

Optima e Perfecta, de modo que não figurassem entre os genótipos com maior massa média de bulbos, confirmando a sua melhor adaptação a cultivos mais antecipados na região.

Apenas seis genótipos se mantiveram no grupo de maior massa média de bulbos na segunda e terceira épocas: os híbridos HB1266, HB2285, HC3119, HC3491 e a variedade OP2931. Quatro genótipos (HC3484, HC3491, LC2951 e LC3153) mantiveram comportamento constante frente às diferentes épocas de cultivo, demonstrando pouca variação diante das alterações das condições ambientais, o que nesse caso não foi vantajoso, pois a massa média apresen- tada por esses genótipos ficou abaixo dos valores alcançados pelos melhores genótipos nas três épocas de cultivo (Tabela 1).

A produtividade de bulbos é uma característica determinante na recomendação de genótipos para uma determinada região ou época de cultivo (Resende $e t$ al., 2007). Para a cultura da cebola, o genótipo ideal é aquele que possui maior produtividade associada a uma maior porcentagem de bulbos pertencentes às classes que recebem melhor remuneração na comercialização (Figueiredo $e t$ al., 2011).

As produtividades obtidas nesse trabalho foram bastante superiores às médias brasileira e paranaense na safra de 2012 (IBGE, 2013). Deve-se levar em conta que em condições experimentais é possível garantir uniformidade no estande e maior nível de controle das condições de manejo, comparativamente às condições de campo em lavouras comerciais. Dessa forma, os elevados valores da produtividade são interpretados como a potencialidade que os genótipos podem atingir em condições semelhantes às experimentais, considerando a população de a $660 \mathrm{mil}$ plantas ha-1.

Houve redução significativa da produtividade dos bulbos, com o avanço da época de cultivo (Tabela 2). Essa redu- 
Tabela 3. Estimativas da heterose de sete híbridos experimentais de cebola, com base em valores médios das linhagens genitoras e híbridos $\mathrm{F}_{1}$ para a massa média de bulbos $(\mathrm{MM})\left(\mathrm{g}_{\text {bulbo }}{ }^{-1} \mathrm{e} \%\right)$ e produtividade de bulbos $(\mathrm{PB})\left(\mathrm{t} \mathrm{ha}^{-1} \mathrm{e} \%\right)$ [heterosis estimates of seven experimental onion hybrids, based on average values of inbred lines and $\mathrm{F}_{1}$ hybrids for the average bulb weight $(\mathrm{MM})\left(\mathrm{g}^{-1}\right.$ bulb and \%) and bulb yield (PB) $\left(\mathrm{t} \mathrm{ha}^{-1}\right.$ and \%)]. Guarapuava, UNICENTRO, 2011.

\begin{tabular}{|c|c|c|c|c|c|c|}
\hline \multirow{3}{*}{ Híbrido } & \multirow{2}{*}{\multicolumn{2}{|c|}{ Genitor }} & \multicolumn{4}{|c|}{ Heterose } \\
\hline & & & \multicolumn{2}{|c|}{ MM } & \multicolumn{2}{|c|}{ PB } \\
\hline & LF & LM & $\left(\mathrm{g}\right.$ bulbo $\left.^{-1}\right)$ & $(\%)$ & $\left(t h^{-1}\right)$ & $(\%)$ \\
\hline HC3490 & LC2873A & LC3068-14 & 26,25 & 46 & 14,7 & 41 \\
\hline HC3489 & LC3031A & LC3068-3 & 23,12 & 35 & 13,8 & 34 \\
\hline HC3485 & LC3031A & LC3153 & 8,92 & 16 & 5,3 & 16 \\
\hline HC3480 & LC3031A & LC3156 & 17,10 & 28 & 10,2 & 27 \\
\hline HC3486 & LC3031A & LC3157 & 24,25 & 40 & 14,7 & 40 \\
\hline НC3483 & LC2873A & LC3153 & 26,40 & 63 & 14,8 & 57 \\
\hline НC3477 & LC2873A & LC3156 & 21,53 & 44 & 11,8 & 39 \\
\hline Média & & & 21,08 & 38 & 12.165 & 36 \\
\hline
\end{tabular}

LF =linhagem feminina; $\mathrm{LM}=$ linhagem masculina. $(\mathrm{LF}=$ female line; $\mathrm{LM}=$ male line $)$.

ção na produtividade ocorreu devido à forte interação genótipos $\mathrm{x}$ ambientes que existe na cultura da cebola (Santos et al., 2004; Leite et al., 2009), em que as diferentes épocas proporcionaram ambientes distintos. Cada genótipo requer um número mínimo de horas diárias de luz (fotoperíodo crítico) para iniciar o processo de enchimento do bulbo (Bosch Serra \& Currah, 2002) o que varia conforme a localidade e a época do ano (Leite et al., 2010).

No plantio tardio (terceira época) o estimulo para a bulbificação ocorreu precocemente, ou seja, antes da planta ter um acúmulo suficiente de fotoassimilados na parte aérea para permitir a otimização do enchimento dos bulbos, o que resulta em bulbos de tamanho menor, proporcionando a redução da produtividade final da cultura (Filgueira, 2005).

Semelhante ao ocorrido para a massa média de bulbos, na primeira época de cultivo, apenas os híbridos comerciais Perfecta, Optima e Buccanneer formaram o grupo que apresentou a maior produtividade (Tabela 2).

O segundo grupo de genótipos mais produtivos na primeira época foi constituído por apenas três híbridos experimentais do grupo Baia (HB1555, HB2285 e HB2572). Os híbridos e linhagens do grupo Crioula apresentaram os piores desempenhos (Tabela 2), uma vez que esses genótipos são derivados de germoplasma adaptado a épocas mais tardias. De maneira geral, houve pior desempenho das linhagens frente aos híbridos, uma vez que a cebola sofre depressão por endogamia, por ser uma espécie alógama (Hallauer \& Miranda Filho, 1995).

Na segunda época de cultivo houve inversão do desempenho relativo de alguns híbridos experimentais (HB1266, HB2285, HC3119, HC3120, HC3481, HC3482, HC3490 e HC3491) e da variedade OP2931, que superaram os híbridos comerciais (Tabela 2). Com isso esses genótipos experimentais de melhor desempenho se mostram promissores para plantios nessa época, pois apresentaram elevadas produtividades, muito superior às médias nacional e estadual.

A terceira época foi considerada a menos promissora para o cultivo e formaram-se apenas dois grupos quanto à produtividade e 22 genótipos foram classificados no grupo de maiores médias, com valores inferiores aos obtidos nas demais épocas, variando dentro da amplitude de 53,1 $\mathrm{tha}^{-1}$ (HC3125) a 36,9 $\mathrm{t}^{-1} \mathrm{a}^{-1}$ (HC3487) (Tabela 2).

Quanto ao comportamento específico de cada híbrido nas três épocas de cultivo, apenas quatro genótipos (HC3484, HC3491, LC2951 e LC3153) mantiveram desempenho constante independentemente da época de cultivo, demonstrando fraca resposta frente às variações ambientais. Esse comportamento não foi vantajoso, pois embora estáveis, as médias foram abaixo dos valores obtidos pelos melhores genótipos nas três épocas de cultivo, impedindo conclusões generalizadas. Todos os demais genótipos apresentaram redução da produtividade com o avanço na época de cultivo (Tabela 2).

Embora na primeira época de cultivo os híbridos comerciais não tenham diferido entre si e tenham sido significativamente superiores aos demais genótipos (Tabela 2), houve inversão de desempenho produtivo dos híbridos Optima e Perfecta em decorrência da diferença na época de cultivo (segunda e terceira épocas), resultante da interação genótipos x épocas, sugerindo que a recomendação desses híbridos para sistemas que visam elevadas produtividades na região de Guarapuava, deve ser restrita à época adequada, nesse caso a primeira época de cultivo.

Para a produtividade de bulbos, apenas $26 \%$ dos genótipos apresentaram índice de confiança geral $\left(\omega_{\mathrm{i}}\right)$ acima de $100 \%$ (Figura 1). Dentre os híbridos experimentais, HB1266 e HB2285 apresentaram os maiores índices de confiança geral para a produtividade ( $\mathrm{Fi}$ gura 1), ou seja, tiveram produtividade de $16,94 \%$ e $16,47 \%$ superior à média dos genótipos nos ambientes avaliados respectivamente, indicando que podem ser considerados estáveis do ponto de vista produtivo (Souza et al., 2013).

Dos híbridos comerciais, apenas o Buccanneer apresentou valores de 


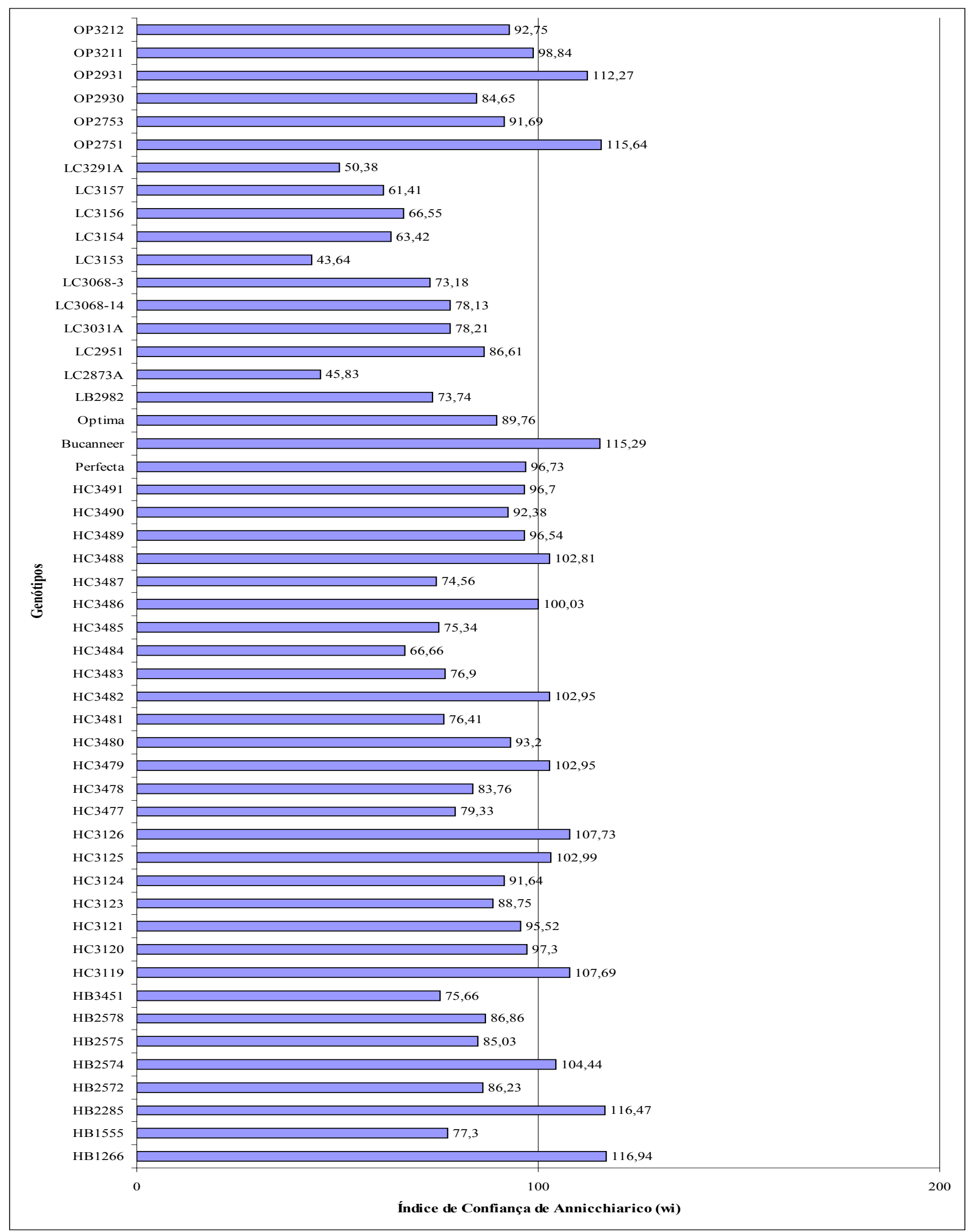

Figura 1. Índice geral de confiança $\left(\omega_{\mathrm{i}}\right)$ de Annicchiarico da produtividade de bulbos de 50 genótipos de cebola cultivados em três épocas [Annicchiarico general confidence index $\left(\omega_{\mathrm{i}}\right)$ of bulb yield of 50 onion genotypes grown in three seasons]. Guarapuava, UNICENTRO, 2011. 
$\omega_{\text {. }}$ acima de $100 \%$, pois em cada uma das épocas as médias de produtividade foram superiores à média geral dos genótipos, revelando a previsibilidade deste híbrido para as condições do experimento. Dentre as variedades OP se destacaram OP2751 e OP2931 (Figura 1).

Os outros genótipos que apresentaram $\omega_{i}$ maior que $100 \%$ foram $\mathrm{HC} 3126$, HC3119, HB2574, HC3125, HC3479, HC3482, HC3488 e HC3486 (Figura 1). Já o genótipo de menor $\omega_{i}$ foi a linhagem LC3153. Os demais genótipos apresentaram $\omega_{\text {i }}$ abaixo de $100 \%$, indicando que o risco de insucesso em se optar um destes genótipos é maior devido os mesmos apresentarem menor estabilidade fenotípica à medida que se altera a data de semeadura.

A heterose média observada para a massa média dos bulbos foi de $38 \%$ $\left(21,08 \mathrm{~g} \mathrm{bulbo}^{-1}\right)$, com variação de $16 \%$ a $63 \%$, sendo que o híbrido que apresentou maior heterose foi HC3483. Outros valores de grande magnitude da heterose foram observados nos híbridos HC3490 e HC3486 (Tabela 3). Relatos de heterose para massa média de bulbos de cebola são encontrados em outros estudos, com variação de $-39,69 \%$ a $6,25 \%$ (Evoor et al., 2007) e -22,99\% a $62,48 \%$ (Abubakar \& Ado, 2008).

A heterose média para a produtividade foi de $36 \%\left(12,2 \mathrm{t} \mathrm{ha}^{-1}\right)$ e variou de $16 \%$ a $57 \%$, sendo que os híbridos mais produtivos (HC3486 e HC3490) apresentaram heterose de 40 e $41 \%$ respectivamente. Faria et al. (2012) observaram valores médios de heterose de $52 \%\left(17,37 \mathrm{t} \mathrm{ha}^{-1}\right)$ para a produtividade de bulbos em Guarapuava-PR. A presença de elevada heterose se torna ainda mais importante quando os genitores (linhagens) são produtivos, pois nem sempre altos valores de heterose são sinônimos de alta produtividade. Os híbridos que apresentaram a maior heterose para massa média dos bulbos foram os mesmos que apresentaram alta heterose para produtividade em decorrência da correlação positiva entre essas variáveis (Faria et al., 2012).

A linhagem LC2873A foi utilizada como genitor feminino nos dois híbridos (HC3483 e HC3490) que apresentaram os maiores valores da heterose, demonstrando assim o potencial de uso dessa linhagem no programa de melhoramento que visa a obtenção de híbridos superiores.

A linhagem LC3031A, genitor feminino do híbrido menos heterótico (HC3485) também participou como linhagem materna do híbrido experimental HC3486 que apresentou a terceira maior heterose dentre os híbridos avaliados. Isso vem confirmar que o valor genético de uma linhagem deve ser avaliado em combinações híbridas, que permite avaliar a capacidade específica de combinação (Faria et al., 2012). A heterose de um dado cruzamento está diretamente relacionada à presença de genes com efeito de dominância e à divergência genética existente entre os genitores (Santos et al., 2011), sendo necessárias diferenças nas freqüências alélicas nos locos envolvidos na expressão do caráter (Hallauer \& Miranda Filho, 1995).

Evoor et al. (2007) observaram que os valores de heterose para produtividade de bulbos em híbridos de cebola variaram de $-30,14 \%$ até $45,31 \%$. Abubakar \& Ado (2008) registraram variação $-72,97 \%$ a $17,26 \%$ nas estimativas da heterose para produtividade de bulbos. Embora no presente estudo não se tenha encontrado estimativas negativas para a heterose da produtividade de bulbos, é possível que elas ocorram. Valores diferentes de heterose refletem o grau de diferenciação das linhagens quanto à capacidade de combinação, em que combinações híbridas com valores de heterose negativos, indicam capacidade de combinação desfavorável no caso da produtividade (Maluf, 2001).

Os híbridos experimentais HB1266 e HB2285 foram considerados estáveis, pois apresentaram os maiores índices de confiança geral para a produtividade. Além disso, a produtividade desses híbridos experimentais na segunda e terceira épocas não diferiu significativamente dos melhores genótipos, o que os torna potencialmente aptos a serem recomendados para essas condições na região de estudo, demandando ainda avaliações complementares.

\section{REFERÊNCIAS}

ABUBAKAR L; ADO SG. 2008. Heterosis of purple blotch (Alternaria porri) resistance, yield and earliness in tropical onions (Allium cepa). Euphytica 164: 63-74.

AGRIANUAL. 2011. Anuário da Agricultura Brasileira. São Paulo: FLP Consultoria e comércio, 2010. 502p.

ANNICCHIARICO P. 1992. Cultivar adaptation and recomendation from alfafa traits in northern Italy. Journal of Genetics and Plant Breeding 46: 269-278.

BOSCH SERRA AD; CURRAH L. 2002. Agronomy of onions. In: RABINOWITCH HD; CURRAH L (eds). Allium crop science: recent advances. Wallingford: CABI Publishing. p. 187-232.

CRUZ CD; CARNEIRO PCS. 2006. Modelos biométricos aplicados ao melhoramento genético. 2 ed. v.2, Viçosa: UFV. 585p.

CRUZ DC. 2007. Programa Genes- Aplicativo computacional em genética e estatística. Viçosa: UFV. 394p.

EVOOR S; GOWDA R; GANGAPPA E; MONOHAR RK. 2007. Heterosis for yield, yield components and quality traits in onion (Allium cepa). Karnataka Journal of Agricultural Sciences 20: 813-815.

FARIA MV; MORALES RGF; RESENDE JTV; ZANIN DS; MENEZES CB; KOBORI RF. 2012. Desempenho agronômico e heterose de genótipos de cebola. Horticultura Brasileira 30: 220-225.

FERREIRA DF. 2000. Sistemas de análises estatísticas 3.1. Lavras: FAEPE/UFLA/PEX.

FIGUEIREDO AST; RESENDE JTV; HUNGER H; PAULA JT; DIAS DM; FARIA MV. 2011. Desempenho de genótipos comerciais de cebola cultivados em diferentes densidades populacionais. Horticultura Brasileira 29: 2265-2272.

FILGUEIRA FAR. 2005. Novo Manual de Olericultura: agrotecnologia moderna na produção e comercialização de hortaliças. Viçosa: UFV. 402p.

HALLAUER AR; MIRANDA FILHO JB. 1995. Quantitative genetics in maize breeding. $2^{\text {nd }}$ ed. Ames: Iowa State University Press. 468p.

IBGE - INSTITUTO BRASILEIRO DE GEOGRAFIA E ESTATÍSTICA. 2013, janeiro. Levantamento sistemático da produção agrícola: pesquisa mensal de previsão e acompanhamento das safras agrícolas no ano civil. Disponível em <file://C:/Documents\%20and\%20Settings/ Usuario/Meus\%20documentos/Downloads/ estat\%C3\%ADstica_cebola_pg38.pdf $>$.

LEITE DL; OLIVEIRA VR; SANTOS CAF; COSTA ND; FONSECA MEN; BOITEUX LS; MELO PE; REIS A; UENO B. 2009. Melhoramento genético de cebola para as condições tropicais e subtropicais do Brasil. Revista Colombiana de Ciencias Hortícolas 3: 18-27.

LEITE DL; OLIVEIRA VR; SANTOS FAC; COSTA ND. 2010. Influência do fotoperíodo e temperatura na bulbificação de cultivares de cebola. Campo \& Negócios 56: 57-59.

MALUF WR. 2001. Heterose e emprego de híbridos $\mathrm{F}_{1}$ em hortaliças. In: NASS LL; VALOIS ACC; MELO IS; VALADARES- 
INGLIS, MC (eds) Recursos genéticos e melhoramento - Plantas. Rondonópolis: Fundação MT. p. 327-356.

MAROUELLI WA; COSTA EL; SILVA HR. 2005. Irrigação da cultura da cebola. Circular Técnica 37 EMBRAPA Hortaliças. 17p. Disponível em <http:/www.cnph.embrapa.br/ paginas/serie_documentos/publicacoes2005/ ct_37.pdf $>$

MAY A; CECÍLIO FILHO AB; PORTO DRQ; VARGAS PF; BARBOSA JC. 2007. Produtividade de híbridos de cebola em função da população de plantas e da fertilização nitrogenada e potássica. Horticultura Brasileira 25: 53-59.
RESENDE GM; CHAGAS SJR; PEREIRA LV. 2003. Características produtivas de cultivares de cebola no sul de Minas Gerais. Horticultura Brasileira 21: 722-725.

RESENDE JTV; PIRES DB; CAMARGO LKP; MARCHESE A. 2007. Desempenho produtivo de cultivares de cebola em Guarapuava, Paraná. Ambiência 3: 193-199.

SANTOS CAF; OLIVEIRA VR; RODRIGUES MA; RIBEIRO HLC; SILVA GO. 2011. Similaridade genética entre cultivares de cebola de diferentes tipos e origens, baseada em marcadores AFLP. Horticultura Brasileira 29: 32-37.

SANTOS GM; BRAZ LT; BANZATTO DA;
DELMANACO JF. 2004. Implicações da interação genótipo $\mathrm{x}$ ambiente em cebola. Horticultura brasileira 22, suplemento CDROM.

SIRTOLI MF; FURLAN L; RODRIGUES JD. 2010. Avaliação de cultivares de cebola para conserva em diferentes épocas de semeadura em Marechal Cândido Rondon-PR. Scientia Agraria Paranaensis 9: 5-14.

SOUZA VF; PARRELLA RAC; TARDIN FD; COSTA MR; CARVALHO JÚNIOR GA; SCHAFFERT RE. 2013. Adaptability and stability of sweet sorghum cultivars. Crop Breeding and Applied Technology 13: 144-151. 